\title{
Understanding the Non-Perturbative Deep-Inelastic Scattering: Instanton-induced Inelastic Dipole-Dipole Cross Section
}

\author{
Edward V.Shuryak and Ismail Zahed \\ Department of Physics and Astronomy \\ State University of New York, Stony Brook, NY 11794-3800
}

(November 5, 2018)

\begin{abstract}
We derive the semiclassical (instanton-induced) contribution to the inelastic cross section of two color dipoles at large $\sqrt{s}$. We study its dependence on the dipole sizes, orientations and most importantly the impact parameter. The inelastic cross section is approximately quadratic in the dipole sizes, and Gaussian-like in the impact parameter with a width of the order of the instanton size. These results are directly relevant to double DIS $\gamma^{*} \gamma^{*}$, as well as $\gamma^{*} \gamma$ and standard DIS $\gamma^{*} h$ at small $\mathrm{x}$ when a real photon and a hadron can be approximated by a dipole. For such cases, with one small dipole scattering on a large dipole, the impact parameter profile exhibits a width of about $1 / 2 \mathrm{fm}$, which is in good agreement with the impact parameter profile recently extracted from DIS HERA data, including diffractive $\gamma^{*} \rightarrow J / \psi$.
\end{abstract}

\section{INTRODUCTION}

\section{A. A puzzling "small gluonic spot"}

Deep Inelastic Scattering (DIS) of leptons on a nucleon is one of the best studied processes in high energy physics, a benchmark for QCD applications since its early days. Still it continues to surprise us, with new data raising yet new questions.

Perturbative (pQCD) treatment of the $Q^{2}$ evolution, first by the OPE methods and moments, and then by the DGLAP evolution equation [1] is now a textbook material, correctly describing the $Q^{2}$ dependence at large enough $Q$. Surprisingly, the non-perturbative aspects of DIS were not much discussed in the literature, and in practice they were always treated as a "phenomenological input" into the DGLAP evolution, usually at some low scale $Q \approx 1 \mathrm{GeV}$.

In the 1970's there was hope that all the glue in the nucleon is basically radiated from valence quarks, in a process described by familiar DGLAP splitting functions. This description implies that the 3 valence quarks in the nucleon should be used as inputs without any $\bar{q} q$ sea or intrinsic glue. However, DIS data, especially from HERA (see e.g. [2]), do not support such views and some intrinsic glue must be present even at the lowest normalization scale, both at large and small $\mathrm{x}$. The physical nature of this intrinsic glue is the main issue to be discussed in this paper.

One important discovery made at HERA relates to diffractive DIS with a surprisingly large cross section (about $10 \%$ of the total cross section). On general grounds one may think that a survival of the nucleon, in spite of a violent DIS collision, means that the gluonic objects hit in the process may be loosely related to the core of the nucleon, or far from its valence quarks.

This discovery also triggered a shift towards treatment of DIS in the nucleon rest frame, in which a virtual photon is substituted by a dipole, frozen in size during its passage through the target. The empirical dependence on the dipole size and energy at low $x$ was discussed by Golec-Biernat and Wusthoff [3], Frankfurt, McDermott and Strikman [4] and others. They all claimed that the dipole cross section shows a particular scaling with dipole sizes, above which the cross section saturates.

The second important discovery came from the analysis of diffractive DIS, such as $\gamma^{*} p \rightarrow J / \psi X p$. In particular, Kopeliovich and his collaborators [5] argued that gluon fluctuations from quarks cannot be described perturbatively and should be restricted to small spots of the order of $0.3 \mathrm{fm}$ in the trasverse direction.

In a paper devoted to global analysis of DIS using nonlinear evolution towards small $x$, Gotsman et al [6] have observed that the input mean square transverse size of the glue $R_{\perp}^{2}$ (at $x \approx 10^{-2}$ ), shows the best fit for its value to be about $3 \mathrm{GeV}^{-2}$, again much smaller than the electromagnetic radius.

In a more recent paper devoted to the analysis of this process by Kowalski and Teaney [7], the dipole model was generalized to local expressions in transverse plane. The impact parameter profile of the glue in the nucleon was found to be small, with an rms width much smaller than the nucleon electromagnetic radius. They also related the small size of the gluonic spot to small nuclear shadowing for gluons. They argued that shadowing is small simply because small spots of glue do not overlap in the impact parameter space. A direct confirmation of such a picture came more recently from $\mathrm{d}$-Au collisions at RHIC. Instead of the widely expected saturation, it was found that even a nucleon going through the diameter of the Au nucleus, with more than a dozen nucleons on its way, experiences only small shadowing corrections to its gluonic structure function that are even overcome by (modest) Cronin effects.

Outside DIS, it is known that soft diffractive hadronic phenomena are related with small-size gluonic objects. For instance, the soft pomeron parameters are related to the small gluonic objects, with the pomeron slope $\alpha^{\prime} \approx$ $1 /(2 \mathrm{GeV})^{2} \approx(0.1 \mathrm{fm})^{2}$ setting the scale. More specifically, it is well established that the pomeron-related form- 
factors are harder than the electromagnetic ones. Those form-factors are completely consistent with the profile $T(b)$ discussed in [7]. In general, the DIS data can be well parameterized by a two-pomeron model (soft plus hard) [8]. Although at large $Q^{2}$ the DIS data show some quantitative differences, we will assume that all diffractive phenomena are of common origin.

In view of all these results, it appears that the current belief - that the gluons are radiated from the valence quarks in a cascade of radiative processes - should be revisited. Indeed, how is it possible that a random radiation process starting with valence quarks within a transverse size of the order of the electromagnetic radius, result in a spot smaller in size than the electromagnetic radius, the spread of the original valence quarks?

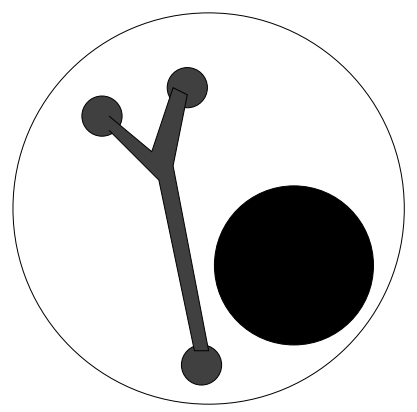

FIG. 1. A schematic view of a nonperturbative glue distribution in a nucleon. The black spot is an instanton.

\section{B. The non-perturbative pomeron and DIS}

After outlining the phenomenological puzzle to be addressed, let us move to its theoretical explanation. In short, we will argue that the small-size gluonic object appearing both in diffractive hadronic and DIS collisions at high energies are instantons (leading to sphaleron production), coherent non-perturbative objects describing large-amplitude fluctuations of the gauge fields in the QCD vacuum. They are dominant for small- $x$ gluons because their field strengths are larger than that of the accompanying valence quarks. A schematic picture of a nonperturbative glue in a nucleon, as seen by a passing dipole, is shown in Fig. 1.

But before we describe this in detail, we first comment on previous important contributions to this field. The non-perturbative approaches to this problem have been sought in the context of the stochastic vacuum model by the Heidelberg group [9]. In this model effective gluonic fields are reduced to Gaussian fluctuations, with "nonperturbative propagators" used for gluon exchanges, and no non-linear terms or interaction vertices. As a result, this model provides a successful account of the constant cross section, but does not account for its growth with energy, since no gluons or other objects can be promptly produced. In this sense, these works focus on the descrip- tion of the constant term $\sigma_{0}$ in the hh cross section

$$
\sigma(s)=\sigma_{0} s^{\Delta} \approx \sigma_{0}+\sigma_{1} \ln s+\ldots
$$

Our discussion below is focused on the $\sigma_{1}$ term. The logarithmic dependence on the energy comes from the longitudinal phase space for the production of QCD sphalerons, which follow the instanton excitation. In this sense, our approach and the one pursued by the Heidelberg group are complementary.

The relevance of instantons to DIS at large $x$ was discussed a decade ago by Balitsky and Braun [10] and also Ringwald and Schrempp [11]. The latter authors have refined the idea in a number of important works, where they focused on specific finite states signatures through an instanton event generator. They also suggested dedicated experiments which were carried at HERA. In these works the focus is on the mechanism in which DIS resolves the instanton, kicking the light quark out of its zero mode. The corresponding expressions are exponentially suppressed at large momentum transfer, e.g. $e^{-Q \rho}$. In our approach the quarks form a frozen dipole, in other words they behave like heavy static quarks and interact with instantons only via its gluonic field. This mechanism is dominant at low $x$. Therefore, our approach at low $x$ complements the approaches pursued at large $x$.

The closest in spirit to our treatment of DIS are recent works by Schrempp and Utterman [12]. In their most recent paper (last citation) a brief reference to Wilson loop scattering through one-instanton is made much along the lines we have advocated in [13] for dipole-dipole scattering. Their starting point is the optical theorem relating the total DIS cross section to the imaginary part of the forward scattering amplitude. However, they appear to use the one-instanton amplitude which is purely real. The one-instanton cannot be cut [13]. Only rescattering, with an instanton in the amplitude and an antiinstanton in the conjugate amplitude with explicit quasielastic or inelastic states crossing the unitarity cut, lead to an imaginary part. Therefore, our results are overall different. In particular, there is no saturation of the dipole cross section $\sigma(\mathbf{d})$ at large dipole size $\mathbf{d}$.

This paper is one in line of a few we have written in the past few years regarding high energy and low angle scattering through instantons. A relation between instantons and diffractive physics (pomerons) was suggested in [14], where in particular a relation between the DIS dipole cross section $\sigma(r)$ were argued to be related to the mean vacuum instanton size $\rho \approx 1 / 3 \mathrm{fm}$ [15]. The importance and the details on how to calculate the instanton-induced quasi-elastic parton-parton and dipole-dipole scattering have been developed in [13], with the resulting small cross section proportional to the instanton diluteness squared. However, if one sums over all gluons radiated from the instanton into the final state, which is equivalent to the production of certain semiclassical gluonic clusters - the QCD sphalerons [16] - the cross section becomes much larger and is proportional to the diluteness parameter in the first power. 
As shown in $[17,18]$, this approach produces reasonable parameters for the soft pomeron. A single sphaleron production gives the $\sigma_{1} \ln s$ term in (1), and its t-channel iteration provides higher powers of the $\ln s$. We have extended such calculations to double-pomeron processes and related properties of QCD sphalerons with clusters and/or identified hadrons produced in this way in [19]. The relation between the so called "color glass condensate" and sphalerons, especially in the early stages of heavy ion collisions, have been extensively discussed in [20].

In this paper we focus on the double DIS process, with two frozen small-size dipoles moving with a speed of light in opposite directions. In this ultimate process, perhaps to be realized at the Next Linear Collider, there is no proton or other hadron in the process, and the non-perturbative glue which generates the dipole-dipole scattering belongs to the wave function of the QCD vacuum itself. One can think of it as zero point oscillations around zero fields, resulting in the usual propagators, and large-amplitude (semiclassically described) fields, representing "tails" of the wave function under the barrier. We will not discuss here any of the the vacuum instanton physics, which can be found e.g. in review [21]. For our purposes it is enough to remind the reader that the diluteness factor $\kappa=n_{\text {ins }} \rho^{4} \approx 10^{-2}$ because the instanton density $n_{\mathrm{ins}} \approx 1 / \mathrm{fm}^{4}$ and the mean size of $\mathrm{QCD}$ instantons is $\rho \approx 1 / 3 \mathrm{fm}$. The diluteness is formally $\sim \exp \left(-2 \pi / \alpha_{s}(\rho)\right)$, which explains why they are invisible in perturbative diagrams. This diluteness of the instanton vacuum leads to a relative diluteness of the semiclassical glue in the proton, and is behind the small shadowing corrections.

In this paper we extend our earlier results to inelastic dipole-dipole scattering, the basic process of great interest to low $x$ DIS. We will show that modulo the enhancement in the overall cross section caused by the production of a sphaleron, our inelastic cross section is in agreement with the quasi-elastic instanton amplitude for dipole-dipole scattering [13]. In particular, the inelastic cross section scales with the squared dipole size for small dipoles, and asymptotes the squared sphaleron (instanton) size for large dipoles.

Even without a calculation, the instanton approach explains why the field is strong and classical, $\approx 1 / g$, and why it appears in small-size spots. Such qualitative discussion was in the literature for decades, see e.g. [22]. The diluteness of the instanton vacuum insures that although there are many partons (dipoles) in a nucleon, presumably only about one may meet the tunneling process active at the moment and near the place of the collision. Phenomenologically, a simple qualitative eikonal estimate in $p p$ and $p \bar{p}$ shows that

$$
\frac{\sigma_{\mathrm{el}}}{\sigma_{\mathrm{in}}} \approx \frac{1-\sqrt{\mathbf{p}}}{1-\mathbf{p}} \approx \frac{1}{4}
$$

at $\sqrt{s} \approx 30 \mathrm{GeV}$. For a Poisson distribution the probability of no collision $\mathbf{p} \approx e^{-\langle S\rangle}$ where $\langle S\rangle \approx 0.8$ is the average number of inelastic pair collisions. This is suggestive of less than one sphaleron per collision at such energies produced. For DIS, with its single small dipole, it can only be a single instanton involved.

The inelastic dipole-dipole cross section has the generic structure,

$$
\sigma_{I N} \approx \ln s \mathbf{d}^{2} \overline{\mathbf{d}}^{2} \rho^{2} n_{\text {ins }}
$$

times a dimensionless function of $\mathbf{d} / \rho$ and $\overline{\mathbf{d}} / \rho$ as well as the impact parameter $\mathbf{b} / \rho$, to be discussed below. This scaling has already been noted in [13] for quasi-elastic processes. Again, the logarithm of the energy comes from the longitudinal phase space of the sphaleron. We recall that the cross section is of first order in the instanton density, not second order and vanishes whenever any of the parameters $\mathbf{d}, \overline{\mathbf{d}}, \rho$ goes to zero.

As discussed in $[17,18]$ and elsewhere, the semiclassical sphaleron production cross section is not yet calculated up to an absolute numerical constant. Therefore we focus on the predicted dependence on the variables in question, the dipole sizes and the impact parameter. Strictly speaking, the dipole-dipole cross section should be used for the double DIS $\gamma^{*} \gamma^{*}$ reactions, which however has not yet been studied in sufficient details. We show however, that the dependence on the dipole size and especially on the impact parameter is in agreement with current HERA data on $\gamma^{*} p$, if we were to think of a nucleon as a color dipole as well.

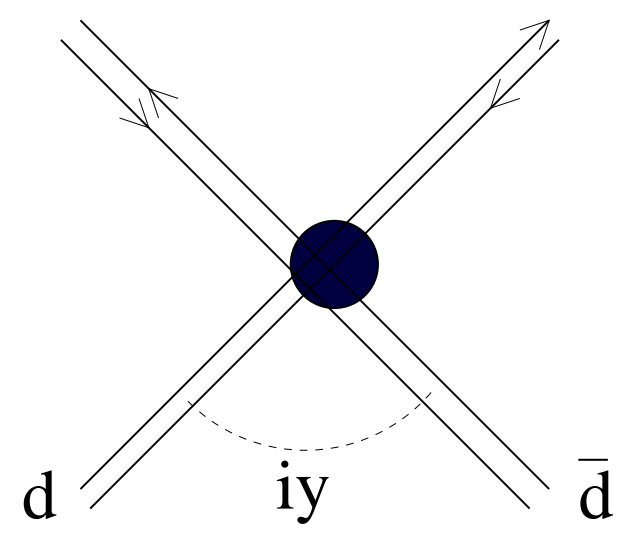

FIG. 2. Two dipoles of sizes $\mathbf{d}, \overline{\mathbf{d}}$ propagate by straight crossing lines, with the Euclidean angle between them being $i y$ where $y$ is the relative rapidity. The centers of these dipoles are also separated by a minimal impact parameter $\mathbf{b}$ (not shown). The dashed circle near the collision point is the instanton, its radius is $\rho$.

\section{DIPOLE-DIPOLE SCATTERING}

In this section we derive the general form of the inclusive dipole-dipole scattering amplitude using QCD sphalerons in for large $\sqrt{s}$ as discussed in $[13,17]$. We 
will briefly recall the dipole-dipole structure through pertinent Wilson lines, and then derive the general form of the inelastic cross section which is our main result. The quasi-elastic cross section with only color-flip in the intermediate state follows by inspection.

\section{A. Eikonalized Dipole-Dipole}

The notations we use and the overall setting of the calculation is explained in Fig.2. Each dipole is represented by a pair of Wilson lines moving by straight lines. The scattering amplitude is proportional to the vacuum expectation value of these 4 Wilson lines. Expansion in the field strength $g A_{\mu}$ would generate the pQCD diagrams. However for instanton fields the expansion is not possible and each quark passing through the instanton has a color rotation of the order one, see [13] for details.

Let $A A$ be the initial color of the dipole and $C D$ its final color. The Wilson loop with open final color for the dipole configuration in the one-instanton background is $[13]$

$$
\begin{aligned}
& \mathcal{W}_{A A}^{C D}(\theta, b)=\cos \alpha_{-} \cos \alpha_{+} \mathbf{1}_{C D} \\
& +i \cos \alpha_{-} \sin \alpha_{+} \mathbf{R}^{a b} \hat{\mathbf{n}}_{+}^{b}\left(\tau^{a}\right)_{D C} \\
& -i \sin \alpha_{-} \cos \alpha_{+} \mathbf{R}^{a b} \hat{\mathbf{n}}_{-}^{b}\left(\tau^{a}\right)_{D C} \\
& +\sin \alpha_{-} \sin \alpha_{+} \mathbf{R}^{a b} \mathbf{R}^{c d} \hat{\mathbf{n}}_{-}^{b} \hat{\mathbf{n}}_{+}^{d}\left(\tau^{c} \tau^{a}\right)_{D C} .
\end{aligned}
$$

We have defined

$$
\begin{aligned}
\alpha_{ \pm} & =\pi\left(1-\frac{\gamma_{ \pm}}{\sqrt{\gamma_{ \pm}+\rho^{2}}}\right) \\
\gamma_{ \pm}^{2} & =\left(y_{4} \sin \theta-y_{3} \cos \theta\right)^{2}+(\mathbf{y} \pm \mathbf{d} / 2)^{2} \\
\mathbf{n}_{+} \cdot \mathbf{n}_{-} & =\left(y_{4} \sin \theta-y_{3} \cos \theta\right)^{2}+\mathbf{y}^{2}-\mathbf{d}^{2} / 4
\end{aligned}
$$

with $\mathbf{n}_{ \pm} \cdot \mathbf{n}_{ \pm}=\gamma_{ \pm}^{2}$. The dipole is sloped at an angle $\theta$ from the $y_{4}$ direction, but otherwise is at an arbitrary location away from the instanton centered at the origin.

The dipole-dipole scattering amplitude with open-color in the final state can be constructed by using two dipole configurations as given by (2) with a relative angle $\theta$. After averaging over the instanton color-orientations, the dipole-dipole amplitude is [13]

$$
\begin{aligned}
& \mathcal{W}_{A A}^{C D}(\theta, b) \underline{\mathcal{W}}_{A^{\prime} A^{\prime}}^{C^{\prime} D^{\prime}}(0,0)= \\
& \frac{2}{N_{c}} \mathbf{W}_{1} \mathbf{1}_{C D} \mathbf{1}_{C^{\prime} D^{\prime}}+\frac{1}{N_{c}^{2}-1} \mathbf{W}\left(\tau^{a}\right)_{D C}\left(\tau^{a}\right)_{D^{\prime} C^{\prime}}
\end{aligned}
$$

The singlet part $\mathbf{W}_{1}$ drops after analytical continuation at large $\sqrt{s}$ and will be ignored. The non-vanishing part of the dipole-dipole cross section is carried by the (octet) part

$$
\begin{aligned}
\mathbf{W} \approx & -\cos \alpha_{-} \sin \alpha_{+} \cos \underline{\alpha}_{-} \sin \underline{\alpha}_{+} \mathbf{n}_{+} \cdot \underline{\mathbf{n}}_{+} \\
& -\sin \alpha_{-} \cos \alpha_{+} \sin \underline{\alpha}_{-} \cos \underline{\alpha}_{+} \mathbf{n}_{-} \cdot \underline{\mathbf{n}}_{-} \\
& +\cos \alpha_{-} \sin \alpha_{+} \sin \underline{\alpha}_{-} \cos \underline{\alpha}_{+} \mathbf{n}_{+} \cdot \underline{\mathbf{n}}_{-} \\
& +\sin \alpha_{-} \cos \alpha_{+} \cos \underline{\alpha}_{-} \sin \underline{\alpha}_{+} \mathbf{n}_{-} \cdot \underline{\mathbf{n}}_{+}
\end{aligned}
$$

where the underlying notation refers to a dipole of size $\overline{\mathbf{d}}$ at angle $\theta=0$ with respect to the $x_{4}$ axis, but again at an arbitrary location away from the instanton located at the origin.

The specificity of (5) is that for a fixed size dipole $\overline{\mathbf{d}}$, as the varying size dipole increases, say $|\mathbf{d}| \gg|\overline{\mathbf{d}}|$ with $\alpha_{-} \approx 0$, it reduces to

$$
\begin{aligned}
\mathbf{W} \approx & -\sin \alpha_{+} \cos \underline{\alpha}_{-} \sin \underline{\alpha}_{+} \mathbf{n}_{+} \cdot \underline{\mathbf{n}}_{+} \\
& +\sin \alpha_{+} \sin \underline{\alpha}_{-} \cos \underline{\alpha}_{+} \mathbf{n}_{+} \cdot \underline{\mathbf{n}}_{-}
\end{aligned}
$$

which is the scattering of a point charge on a fixed size dipole $\overline{\mathbf{d}}$. This is a property of the unexpanded sine and cosine contributions (re-summed gluon lines) stemming from the unitary character of the Wilson lines. It will prove important below for our discussion on asymptotia.

\section{B. Inelastic Dipole-Dipole Cross Section}

In terms of (5) the inelastic cross section for dipoledipole scattering through sphalerons follow the general construction developed in [17] for parton-parton (chargecharge) scattering. To leading logarithm accuracy, the result in Minkowski space is

$$
\begin{aligned}
& \sigma_{I N}(s) \approx \ln \operatorname{Im} \sum_{C D, \underline{C D}} \frac{1}{(2 \pi)^{6}} \int d Q^{2} d q_{1 \perp} d q_{2 \perp} \\
& \times \int d z d \dot{I} d \dot{I}^{\prime} e^{i Q z+i S(\dot{I})-i S\left(\dot{I}^{\prime}\right)+i S\left(\dot{I}, \dot{I}^{\prime}, z\right)} \\
& \times \int d x_{-} d x_{\perp} d y_{+} d y_{\perp} e^{-i q_{1 \perp} x_{\perp}-i q_{2 \perp} y_{\perp}} \\
& \times \mathcal{W}_{A A}^{C D}\left(\infty, x_{-}, x_{\perp}\right) \underline{\mathcal{W}} \underline{\underline{A A}}\left(y_{+}, \infty, y_{\perp}\right) \\
& \times \int d x_{-}^{\prime} d x_{\perp}^{\prime} d y_{+}^{\prime} d y_{\perp}^{\prime} e^{-i q_{1 \perp} x_{\perp}^{\prime}-i q_{2 \perp} y_{\perp}^{\prime}} \\
& \times \mathcal{W}_{A A}^{C D *}\left(\infty, x_{-}^{\prime}, x_{\perp}^{\prime}\right) \underline{\mathcal{W}} \underline{\underline{A A}} *\left(y_{+}^{\prime}, \infty, y_{\perp}^{\prime}\right),
\end{aligned}
$$

where the collective integrations $z, \dot{I}, \dot{I}^{\prime}$ correspond to localized chromomagnetic field in space-time. The appearance of $\ln s$ underlines the fact that the integrand in (7) involves only $Q^{2}$ which is the transferred mass in the inelastic half of the forward amplitude, and $q_{1,2} \perp$ which are the transverse transferred momenta through the dipole form-factors.

We now elect to evaluate (7) in Euclidean space by interpreting the localized fields as singular gauge fields (sphaleron) that maximize the partial scattering amplitude in $Q^{2}$, and the external dipole amplitudes with (4-5) with the identification $\theta \leftarrow i \ln s$. The result is

$$
\sigma_{I N}(s) \approx \int \frac{d \mathbf{q}}{(2 \pi)^{2}}\left|\int d \mathbf{b} e^{-i \mathbf{q} \cdot \mathbf{b}} \mathbf{F}(s, \mathbf{b})\right|^{2}
$$

where 


$$
\mathbf{F}(s, \mathbf{b})=i \sqrt{\kappa \ln s} \frac{4}{\left(N_{c}^{2}-1\right)} \frac{\mathbf{d} \cdot \overline{\mathbf{d}}}{8 \pi \rho^{2}} \mathbf{I}(\mathbf{d} / \rho, \overline{\mathbf{d}} / \rho, \mathbf{b} / \rho)
$$

defined via the dimensionless function of the three $2-\mathrm{d}$ vector variables

$$
\begin{aligned}
& \mathbf{I}(\mathbf{d} / \rho, \overline{\mathbf{d}} / \rho, \mathbf{b} / \rho) \equiv \frac{1}{\rho^{2}} \int d \mathbf{R} d x_{-} d y_{+} \\
& \times\left(\frac{c_{-} s_{+} \underline{c}_{-} \underline{s}_{+}}{\gamma_{+} \underline{\gamma}_{+}}+\frac{s_{-} c_{+} \underline{s}_{-} \underline{c}_{+}}{\gamma_{-} \underline{\gamma}_{-}}+\frac{c_{-} s_{+} \underline{s}_{-} \underline{c}_{+}}{\gamma_{+} \underline{\gamma}_{-}}+\frac{s_{-} c_{+} \underline{c}_{-} \underline{s}_{+}}{\gamma_{-\underline{\gamma}_{+}}}\right)
\end{aligned}
$$

after $(\mathbf{b} \rightarrow-\mathbf{b}, \mathbf{d} \rightarrow \overline{\mathbf{d}})$ symmetrization, and rotation back to Minkowski space in the large $\sqrt{s}$ limit. The sine $s_{ \pm}$and cosine $c_{ \pm}$functions in (10) are defined with arguments $\alpha_{ \pm}$, and similarly for $\underline{\alpha}$ with the substitution $\gamma \rightarrow \underline{\gamma}$, where

$$
\begin{gathered}
\gamma_{ \pm}^{2}=x_{-}^{2}+(\mathbf{R}+\mathbf{b} / 2 \pm \mathbf{d} / 2)^{2} \\
\underline{\gamma}_{ \pm}^{2}=y_{+}^{2}+(\mathbf{R}-\mathbf{b} / 2 \pm \underline{\mathbf{d}} / 2)^{2} .
\end{gathered}
$$

The contributions arising from (10) is mostly through the electric dipoles, and vanish for zero size dipoles. The magnitic contribution is of the form $\mathbf{d} \times \overline{\mathbf{d}}$ and drops under symmetrization. The quasi-elastic cross section with only color-flip in the intermediate states is due to the instanton-antiinstanton as opposed to the sphaleron configuration used here. It is readily checked that it follows from (8) through the substitution $\kappa \ln s \rightarrow \kappa^{2}$.

\section{Eikonal Unitarization}

The inelastic cross section (8) can be readily rewritten as

$$
\sigma_{I N}(s) \approx \int d \mathbf{b}|\mathbf{F}(s, \mathbf{b})|^{2}
$$

which allows $\mid \mathbf{F}\left(s,\left.\mathbf{b}\right|^{2}\right.$ to be interpreted as the absorption probability at fixed impact parameter and energy ${ }^{1}$. In the present case, the absorption probability grows like $\ln s$ with large $\sqrt{s}$ and therefore upsets unitarity constraints (albeit logarithmically). A simple way to fix this is by using the standard eikonal (s-channel) unitarization, i.e.

$$
|\mathbf{F}(s, \mathbf{b})|^{2} \rightarrow 1-e^{-|\mathbf{F}(s, \mathbf{b})|^{2}}
$$

which yields the eikonalized cross sections

$$
\begin{aligned}
& \sigma_{T O T}(s) \approx 2 \int d \mathbf{b}\left(1-e^{-\frac{1}{2}|\mathbf{F}(s, \mathbf{b})|^{2}}\right) \\
& \sigma_{E L}(s) \approx \int d \mathbf{b}\left|1-e^{-\frac{1}{2}|\mathbf{F}(s, \mathbf{b})|^{2}}\right|^{2} \\
& \sigma_{I N}(s) \approx \int d \mathbf{b}\left(1-e^{-|\mathbf{F}(s, \mathbf{b})|^{2}}\right) .
\end{aligned}
$$

The total cross section in (14) saturates when the distance between the dipoles (impact parameter) exceeds the sphaleron size $\rho$.

\section{The dependence on the dipole size and impact parameter}

If the dipoles sizes are small, one expects the dipole approximation to hold and the cross section to be proportional to $\mathbf{d}^{2}$ or $\overline{\mathbf{d}}^{2}$. Such factors are already included in the definition of the function $\mathbf{F}(9)$, and thus one expects a smooth limit of $\mathbf{I}(\mathbf{d} / \rho, \overline{\mathbf{d}} / \rho, \mathbf{b} / \rho)$ when either of the dipoles, or both go to zero. A look at its definition confirms that it is indeed the case.

Now let us qualitatively discuss the $\mathbf{b}$-dependence. One naturally expects a maximum at $\mathbf{b}=0$, so at small impact parameters $\mathbf{I}(\mathbf{b})=\mathbf{I}(\mathbf{b}=0)\left(1-\right.$ const $\left.^{2} / \rho^{2}\right)$. At large $\mathbf{b}$ one expects a power decrease. Indeed, if $\mathbf{b} \gg \rho$ one may think that the main contribution to the cross section comes from the instanton far from each of the lines. In such a case all $\gamma_{ \pm} \approx \mathbf{b} \gg \rho$ are large compared to $\rho$ and the arguments of all trigonometric functions in (10) are small $\alpha_{ \pm} \approx \rho^{2} / 2 \gamma_{ \pm}^{2}$, so that all cosines and sines can be replaced by either 1 or their arguments. Physically this corresponds to a single-gluon exchange between each dipole and the instanton. As a result, our 4-dimensional integral over $x_{-}, y_{+}, R_{x}, R_{y} \approx \mathbf{b}$ will have an integrand $\approx 1 / \gamma^{6}$ and is therefore convergent, producing $\mathbf{I}(0,0, \mathbf{b} / \rho) \sim \rho^{2} / \mathbf{b}^{2}$ with a positive coefficient.

Let us now discuss the issue quantitatively. In our preceding papers, see for instance [19], we have argued that (unlike many other instanton applications) the modification of the instanton profile is numerically important for high energy collisions. The dipole forces at large distances are of course true for massless gluons only and are ultimatly absent in confining theories. A phenomenological way to enforce this was discussed in [19]. In Fig. 3 we show how two selections of cutoffs on the range of the instanton field, with

$$
f_{1}=e^{-0.5 \gamma} \quad f_{2}=e^{-0.08 \gamma^{2}}
$$

correspond to the cross section of double-pomeron pp events from WA102 experiment at CERN. The modification function enters all the arguments of the trigonometric function in $\mathbf{I}$ (10) through the substution $\alpha \rightarrow \alpha f$ with the pertinent $\gamma$ arguments. Note that the instanton size is kept fixed at $\rho=1 / 3 \mathrm{fm}$. The inclusion of a realistic size distribution would somewhat change the small and large $t$ tails.

\footnotetext{
${ }^{1}$ With the addition of the constant part as in (1).
} 


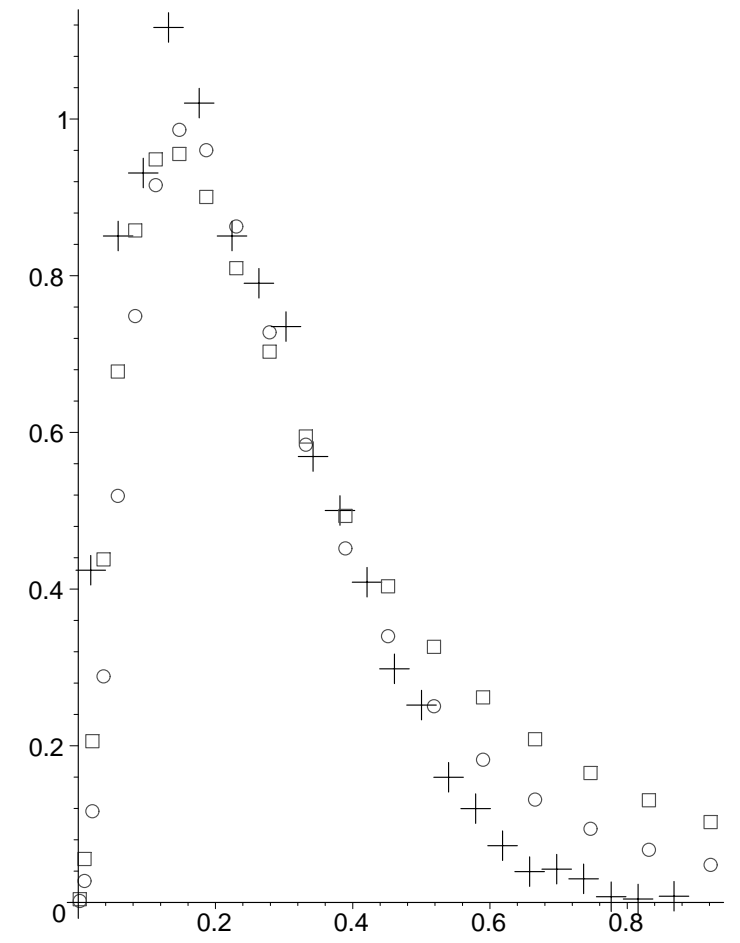

FIG. 3. Instanton shape modification compared to WA102 experimental data (crosses, no error bar shown) on the momentum transfer $t$ (the same for both protons). The squares and circles correspond to the instanton form-factor to the fourth power, with the cutoffs $f_{1,2}(15)$, respectively. The cutoffs produce the dip at small $t$ seen in the data.

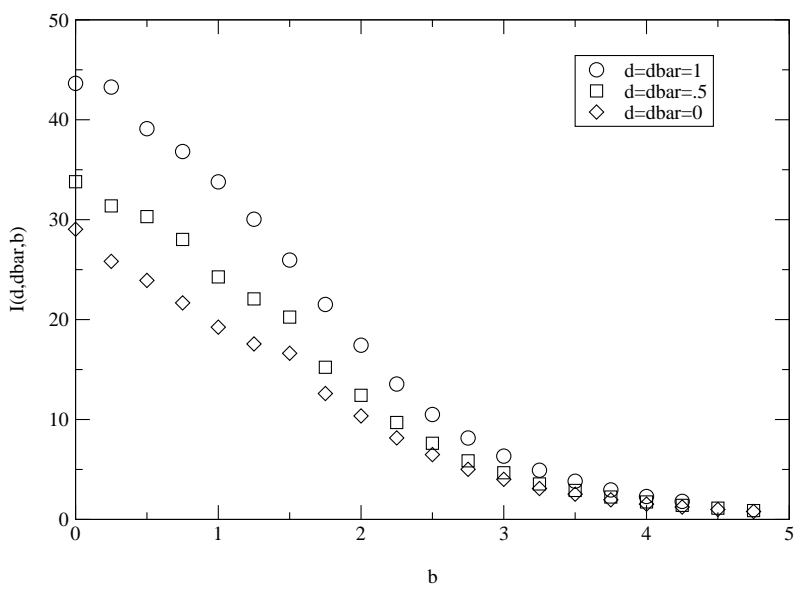

FIG. 4. Dependence of the function $\mathbf{I}(\mathbf{d} / \rho, \overline{\mathbf{d}} / \rho, \mathbf{b} / \rho)$ defined in (10) on its last argument, the impact parameter $\mathbf{b}$. The case shown is for all 3 vectors in the same plane and for equal dipole sizes (in units of the instanton size).

Now we turn to a more quantitative discussion of (10) with the cutoffs (15). In Fig. 4 we show the b-dependence of the pertinent integral, for few values of the dipole sizes. Note also, that if the dipoles are of different sizes, the largest completely dominates. The calculations were carried out using Monte-Carlo with few checks through Mathematica.

In Fig. 5 we show the value of this function when $\mathbf{b}=0$ and both dipoles with equal size. There is almost no dependence on $\mathbf{d}, \overline{\mathbf{d}}$ when the dipoles are less than about $\rho / 2$. The dependence appears when the dipole sizes are comparable to $\rho$, but desappears again when $\mathbf{d}, \overline{\mathbf{d}} \gg \rho$. Remarkably, a much weaker dipole size dependence is found when only one of the dipole size is varied while the other is fixed. For example, if $\overline{\mathbf{d}}=\rho$ the dependence on $\mathbf{d}$ is of the order of few percents in the entire range of the dipole size variation, from small to large.

Finally, we note that the numerical value of the dimensionless function $\mathbf{I}$ is not of the order of 1 but of the order of 20, which is large enough to compensate for a square root of the instanton diluteness. This shows that in a relevant volume near the dipole collision point there is about 1 instanton in the QCD vacuum, and so the cross section is not really suppressed.

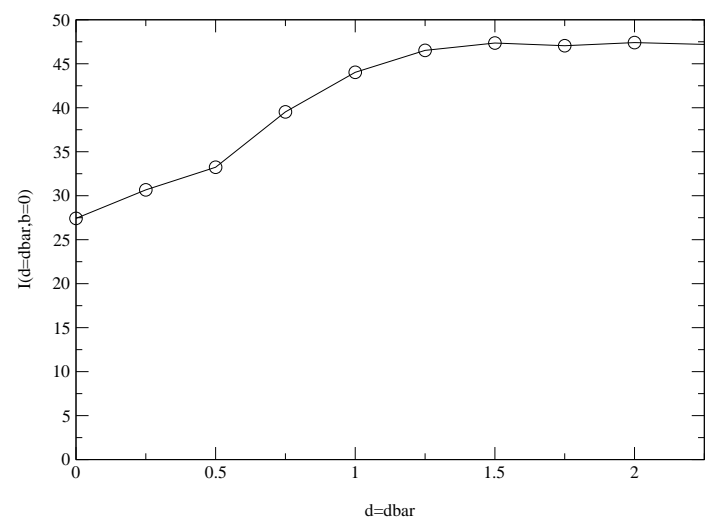

FIG. 5. Dependence of the function $\mathbf{I}(\mathbf{d} / \rho, \overline{\mathbf{d}} / \rho, \mathbf{b} / \rho)$ defined in (10) at $\mathbf{b}=0$ on the dipole size $\mathbf{d} / \rho=\overline{\mathbf{d}} / \rho$.

\section{DIPOLE-DIPOLE CROSS SECTION AND DIS PHENOMENOLOGY}

The main point of this paper is that the glue on which DIS is made is basically from the vacuum, one does not need a nucleon or other quark states. In principle, one can directly compare our results with the experimental data for $\gamma^{*} \gamma^{*}$ collisions. We know rather well how a photon becomes a frozen $\bar{q} q$ dipole. It is well described by a simple loop diagram ${ }^{2}$, see e.g. [4]. The size of a

\footnotetext{
${ }^{2} \mathrm{By}$ the way, the vector current is a special case, in which all non-perturbative corrections tend to cancel to a high accu-
} 
small dipole is related to $Q^{2}$ by $d \approx \pi / Q$.

For sufficiently large $Q^{2}$ and $\bar{Q}^{2}$ the dipole sizes are small compared to $\rho$ and one may set ${ }^{3} \mathbf{d}=\overline{\mathbf{d}}=0$ in our function $\mathbf{I}$ (the lowest set of points in Fig. 4). Note that the impact parameter profile is quite narrow, with a width of about the instanton size.

Unfortunately, past LEP experiments had very small acceptance for such events and had very small statistics. The diffractive events, such as $\gamma^{*} p \rightarrow J / \psi X$, have not been yet studied well enough to test our predictions. There are of course much better DIS data on a nearreal photon, $\gamma^{*} \gamma$, from LEP experiments. Those correspond to the asymmetric case with one small and one large dipole, which is close to the upper set of points in Fig. 4.

The best DIS data are from HERA, for the scattering on a proton $\gamma^{*} p$. The observed dependence of $\sigma_{\gamma * p}$ on $\mathbf{d}$ is a matter of ongoing debate. The original model by GolecBiernat and Wusthoff [3] assumes that it grows as $\sigma_{\gamma * p} \approx$ $\mathbf{d}^{2}$ at small $\mathbf{d}$ and then saturates, at some constant cross section $\sigma_{0} \approx 20 \mathrm{mb}$. Alternative parameterizations of the dipole cross section were also considered, e.g. in [4]. A simultaneous fit to DIS and diffractive $\gamma^{*} p \rightarrow J / \psi+\ldots$ in [7] resulted in different conclusions: (i) there is more or less quadratic dependence on the dipole size $\sigma_{\gamma * p} \approx \mathbf{d}^{2}$ for all sizes with no need for saturation or unitarization; (ii) The b-dependence is given by a function $T(\mathbf{b})$ of roughly Gaussian shape with an rms radius $\mathbf{b}_{\mathrm{rms}}=0.4-$ $0.5 \mathrm{fm}$.

Strictly speaking we do not know if a proton can be seen as a dipole (or a distribution of dipoles). One may still try to use our calculation, thinking about the the nucleon as a single large dipole $(\overline{\mathbf{d}} \approx \rho)$. If so, our results qualitatively agree with Kowalski and Teaney recent observations as mentioned above. In particular, the dependence on the dipole sizes is quadratic without saturation and the width of the "gluonic spot" we predict turns out to be also about $1 / 2 \mathrm{fm}$. We plan to do a more specific model analysis for the proton and carry a detailed discussion of DIS and diffractive DIS in a separate publication.

\section{ACKNOWLEDGMENTS}

This work was supported in parts by the US-DOE grant DE-FG-88ER40388. We thank H. Kowalski, D.

racy, as studies of correlations functions deduced from $e^{+} e^{-}$ and $\tau$ decays indicate, see [22]. This is not the case for scalar or pseudoscalar "photons", if Nature were to provide them.

${ }^{3}$ We recall that the large- $Q$ or small-d dependence is given by DGLAP evolution. We are only discussing the nonperturbative aspects of the problem, ignoring radiative corrections. Our results should be treated as the "initial conditions" for DGLAP at the semi-hard scale $Q \approx 1 \mathrm{GeV}$.
Teaney and G. Papp for valuable discussions, and A. Mueller for attracting our attention to the impact parameter issue in diffractive DIS. We also thank E. Levin, M. Lublinsky and F. Schrempp for helpful correspondence.

[1] V.N. Gribov, L.N. Lipatov, Sov. J. Nucl. Phys. 15 (1972) 438; G. Altarelli and G. Parisi, Nucl. Phys. B126 (1977) 298.

[2] D. Groom et al., Eur. Phys. J. C15 (2000) 1.

[3] K. Golec-Biernat and M. Wusthoff, Phys. Rev. D59 (1999) 014017; D60 (1999) 114023.

[4] L. Frankfurt, M. McDermott and M. Strikman, JHEP02 (1999) 002.

[5] J. Hufner, Y. P. Ivanov, B. Z. Kopeliovich and A. V. Tarasov, Phys. Rev. D62 (2000) 094022.

[6] E. Gotsman, E. Levin, M. Lublinsky and U. Maor, Eur. Phys. J. C27 (2003) 411.

[7] H. Kowalski and D. Teaney, hep-ph/0304189.

[8] A. Donnachie and P. Landshoff, Phys. Lett. B296 (1992) 227; B437 (1998) 408.

[9] H. G. Dosch, V. I. Shevchenko and Y. A. Simonov, Phys. Rept. 372 (2002) 319; A.I. Shoshi, F.D. Steffen and H.J. Pirner, Nucl. Phys. A709 (2002) 131.

[10] I. I. Balitsky and V.M. Braun, Phys. Lett. B314 (1993) 237; Phys. Lett. B346 (1995) 143;

[11] A. Ringwald, F. Schrempp, hep-ph/9411217; Phys. Lett. B438 (1998) 217; Phys. Lett. B503 (2001) 331.

[12] F. Schrempp and A. Utermann, Phys. Lett. B543 (2002) 197; hep-ph/0207052; hep-ph/0301177.

[13] E. Shuryak and I. Zahed, Phys. Rev. D62 (2000) 085014.

[14] E. V. Shuryak, Phys. Lett. B486 (2000) 378.

[15] E. V. Shuryak, Nucl. Phys. B203 (1982) 116.

[16] D. M. Ostrovsky, G. W. Carter and E. V. Shuryak, Phys. Rev. D66 (2002) 036004; R. Janik, E. Shuryak and I. Zahed, Phys. Rev. D67 (2003) 014005.

[17] M. Nowak, E. Shuryak and I. Zahed, Phys. Rev. D64 (2001) 034008.

[18] D. Kharzeev, Y. Kovchegov and E. Levin, Nucl.Phys. A690 (2001) 621.

[19] E. Shuryak and I. Zahed, hep-ph/0302231.

[20] E. V. Shuryak, Nucl. Phys. A715 (2003) 289; Nucl. Phys. A717 (2003) 291.

[21] T. Schafer and E. V. Shuryak, Rev. Mod. Phys. 70 (1998) 323.

[22] E. V. Shuryak, Rev. Mod. Phys. 65 (1993) 1. 